\title{
A Process-Model Account of Task Interruption and Resumption: When Does Encoding of the Problem State Occur?
}

\author{
Dario D. Salvucci \\ Drexel University \\ Philadelphia, PA
}

\author{
Christopher A. Monk \\ George Mason University \\ Fairfax, VA
}

\author{
J. Gregory Trafton \\ Naval Research Laboratory \\ Washington, DC
}

\begin{abstract}
Memory for goals theory (Altmann \& Trafton, 2002) describes how people suspend and resume an interrupted task by encoding, or rehearsing, the current problem state at the point of interruption and recalling this state after the interruption. In this work we investigated the timing of the encoding process, attempting to determine the most likely strategies for when to perform encoding of interrupted problem state. We examined several candidate encoding strategies and developed computational cognitive models to represent each strategy, embedding the models into a larger model of behavior in a interruption-tracking task. Comparison of the model simulations with recent empirical data suggests that encoding of problem state occurs for a short time at the start of the interruption period and is performed concurrently with the interrupting task.
\end{abstract}

\section{INTRODUCTION}

Interruptions represent a ubiquitous phenomenon in our everyday world: While performing some primary task, a person is interrupted and must engage in a secondary task before resuming the primary task. Recent studies have aimed to better understand how we manage task interruptions in situations and environments as diverse as human-computer interaction (e.g., Bailey \& Iqbal, 2008; Cutrell, Czerwinski, \& Horvitz, 2000), aviation (e.g., Dismukes, Young, \& Sumwalt, 1998; Latorella, 1999), and emergency room care (e.g., Chisholm et al., 2001) to name a few. These studies typically focus on understanding how varying factors (e.g., interruption timing or interrupting task type) may differentially affect one's ability to resume and perform in the primary task.

One current theory, memory for goals (Altmann \& Trafton, 2002), describes how the suspension and resumption of a task during an interruption can be best understood in the context of human memory processes. According to this theory, every task has associated information that must be stored in memory during the interruption and recalled from memory after the interruption. This information, which they referred to as part of the goal and which we call the problem state (or problem representation: Borst \& Taatgen, 2007), can be thought of as the mental "scratchpad" used by the current task to carry over short-lived information from one stage of processing to the next (e.g., noting the two addends while solving an addition problem like " $3+2=+$ "). According to memory for goals, this information undergoes a process of encoding: mentally focusing on the information until it can be readily recalled after interruption. The processes used in the memory for goals theory have accounted for a number of phenomena in the task-switching and interruption literature (e.g., Altmann \& Gray, 2007, 2008) and several recent studies have also bolstered the empirical support for the theory (e.g., Hodgetts \& Jones, 2006; Li et al., 2008; Monk, Boehm-Davis, \& Trafton, 2004; Monk, Trafton, \& Boehm-Davis, 2008; Trafton, et al., 2003).

In this paper we develop a process model of interruption and resumption, and at the same time investigate the question of when exactly does problem-state encoding take place in the course of interruption. The original memory for goals theory specified a general process model of encoding and retrieval that incorporated one particular strategy of encoding, namely to encode goal information a few times just before starting the interrupting task. At the same time, we could imagine a number of other candidate strategies whereby encoding occurs before and/or during interruption and requires varying amounts of time and potentially concurrent execution with the interrupting task. In this work we posit a number of candidate encoding strategies, build computational cognitive models to represent each strategy, and compare the resulting behaviors of these models to recent empirical data (Monk, Trafton, \& Boehm-Davis, 2008) to attempt to clarify when encoding occurs in the interruption process.

\section{MEMORY FOR GOALS AND EFFECTS OF INTERRUPTION DURATION AND DEMAND}

Memory for goals (Altmann \& Trafton, 2002) posits that task suspension centers on memory encoding and retrieval of the current goal, which can be thought of as the problem-state information related to the current task (Borst \& Taatgen, 2007). Memory for goals theory is rooted in the activation model of memory in the ACT-R cognitive architecture (Anderson et al., 2004). According to memory for goals, the time required to resume the suspended goal after an interruption is directly related to its level of activation: Goals that have been suspended for longer periods will have decayed to lower activation levels, and therefore will take longer to retrieve and resume. In addition, the model's strengthening constraint indicates how encoding (i.e., rehearsal) before or during the interruption can help strengthen the suspended task state's activation level, therefore diminishing the level of decay. A consequence of the strengthening constraint is that if the interruption task is demanding of cognitive resources such that rehearsal of the suspended task goal is inhibited, then greater decay effects should be evident. 
These effects of interruption duration (shorter or longer) and demand (more or less demanding secondary task) were recently tested in a set of experiments by Monk, Trafton, and Boehm-Davis (2008). The focus of our work here is on Experiment 3, in which they tested these effects using a VCR programming task as the primary task. Participants performed the VCR task while being interrupted every five seconds for durations of 3,8 , or 13 seconds. Three different secondary (interrupting) tasks were included to test the strengthening constraint's predictions of the effects of rehearsal on task resumption: a no-task interruption, during which participants simply waited out the interruption period (providing unlimited opportunity to rehearse); a pursuit-tracking task as the intermediate-demand condition; and an "n-back" task as the high-demand condition. The results showed that greater interruption task demand produced increasingly longer resumption lags, and that even with uninhibited rehearsal availability in the no-task condition, some evidence of a interruption duration (decay) effect persisted. In this paper we focus on modeling the no-task and tracking-task conditions, the results of which are shown in Figure 1.

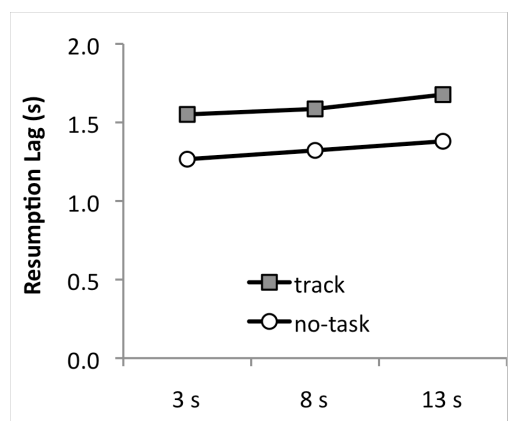

Figure 1: Experiment 3 results from Monk et al. (2008).

\section{PROCESS MODELS OF INTERRUPTION AND PROBLEM-STATE ENCODING}

We first set out to explore how memory for goals and the effects of interruption duration and demand above can be realized as a computational process model. Specifically, while memory for goals posits that encoding and decay are critical for understanding how people resume interrupted tasks, the original work described a single method of encoding (namely to rehearse a few times before interruption). There are, however, a number of other reasonable strategies that people might utilize for encoding. Below we propose several candidate encoding strategies and develop computational models that represent each strategy. We then simulate the strategies and compare the results to Monk et al. (2008) results above, trying to better understand which strategies may provide a better explanation of the empirical data.

\section{Modeling Framework}

The modeling framework that we use to develop our process representations is the ACT-R cognitive architecture combined with extensions that have come out of several recent research efforts. ACT-R (Anderson et al., 2004) serves as both the underlying psychological theory and the computational framework in which to specify and simulate cognitive models. ACT-R separates knowledge into two components: declarative knowledge that represents general facts and current goals, and procedural knowledge that represents behavioral skill in terms of rule firings that produce observable behavior. Critically for our purposes here, the declarative component specifies how memory elements, chunks, strengthen and decay with rehearsal or lack of use. The procedural component allows us to create rule-based skills that embody not only the skills for the primary and secondary tasks but also the skills needed to encode and retrieve goals in the interruption process.

Two recent efforts extending the ACT-R architecture relate directly to our approach to addressing task interruption. First, the theory of threaded cognition (Salvucci \& Taatgen, 2008) posits how two or more ACT-R models can execute concurrently in a multitasking situation - that is, manage the resources between tasks and interleave task steps such that both tasks progress toward completion, often with very efficient execution due to parallelism among resources. Second, the theory of time estimation (Taatgen, van Rijn, \& Anderson, 2007) dictates how a person estimates short time intervals while performing other tasks. Both of these components are critical for our models, as we describe shortly.

An additional research effort related to our own centers on the role of problem state when performing multiple tasks. Borst and Taatgen (2007) investigated how two tasks that require separate problem states can experience multitasking interference, such that the representations need to be swapped out for an interruption and swapped back in after the interruption. It is this problem state (stored in ACT-R's "imaginal" buffer) that is a critical memory chunk for task switching: In the context of the memory-for-goals theory, it is the problem-state memory chunk that must be encoded during an interruption and retrieved after the interruption.

\section{Basic Model and Candidate Encoding Strategies}

Using this framework, the basic process model for problem-state encoding is a straightforward interpretation of the ACT-R architecture and the understanding of problem representations. When a primary task is interrupted, and this task has a problem state that must be saved, the model initiates the encoding process that performs repeated retrievals of the problem state in declarative memory. The core process needs only two procedural steps (production rules in ACT-R), one that initiates the memory retrieval and another that "harvests" the result of this retrieval. As dictated by ACT-R memory processes, the repeated retrievals amount to rehearsal of this memory chunk which increase activation of that memory and result in faster subsequent retrievals. When this encoding process terminates, the problem state has been strengthened enough that it can be readily retrieved after interruption, allowing the primary task to resume successfully.

This description of the basic encoding process, however, does not dictate when to initiate and terminate the encoding process. We examined several possible candidate strategies 
that dictate when people encode before or during a task interruption, and developed a model of each strategy for purposes of evaluating and comparing them:

S1: Encode during the entire interruption. This strategy makes maximum use of encoding, potentially at the expense of secondary-task performance.

S2: Encode for $n$ seconds, concurrently with the secondary task and starting at the onset of the interruption. This strategy assumes that people encode only for a short interval of time, utilizing ACT-R's timing mechanism to time the duration of the interval. Past results have shown that people do perform explicit rehearsal during the secondary task (Trafton et al., 2003).

S3: Encode until retrieval takes no more than $n$ seconds, concurrently with the secondary task and starting at the onset of the interruption. This strategy assumes that people encode until the problem state can be retrieved sufficiently quickly (again using the time estimation mechanism), performing however many retrievals are needed to achieve this.

S4: Encode for $n$ seconds prior to the interruption, ending at the onset of the interruption. This strategy requires some prediction of when the interruption will occur, stopping at some time interval before the interruption to allow for sufficient time for encoding. Of course, such prediction may not be possible in general; however, in the Monk et al. (2008) Experiment 3, interruptions occurred at regular 5-second intervals, and thus we include S4 as another possible encoding strategy.

S5: Encode for $n$ retrievals prior to the interruption, ending at the onset of the interruption. This strategy, first used by Altmann and Trafton (2002), allows for a few retrievals immediately before the start of interruption.

The timing of these encoding strategies is depicted in Figure 2. It should be noted that $\mathrm{S} 1, \mathrm{~S} 2$, and $\mathrm{S} 3$ all require encoding to be concurrent with (i.e., executed in parallel with) the secondary task. (Note that S4 and S5 are performed during a self-imposed interruption lag between the primary and secondary tasks.) The concurrent execution of the encoding and secondary tasks is realized by threaded cognition, which interleaves the cognitive steps of the encoding task with those of the encoding task.

\section{Model of Monk et al. (2008) Experiment 3}

We developed a computational ACT-R model of the interruption task studied by Monk et al. (2008). The model included only those components necessary to generate predictions of the relevant dependent measure, namely resumption lag, or time to resume the primary task after conclusion of the interruption. To this end, the primary task (the VCR task) was not modeled in detail; it was sufficient simply to create a problem-state representation as a declarative memory chunk to be saved during interruption and retrieved after interruption. The model of the secondary (interrupting)

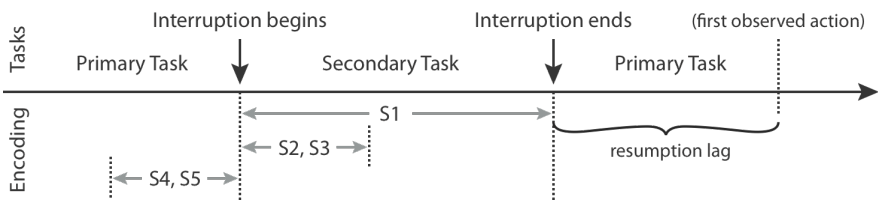

Figure 2: Encoding strategies during interruption.

task was more critical for our purposes and thus more detailed. The tracking task interface and the model of tracking behavior were imported directly from earlier work (Salvucci \& Taatgen, 2008), with a slight modification to mimic the Monk et al. task (a randomly-moving target with the cursor moved to follow the target). The model of the no-task condition trivially waited until the end of the interruption.

For a single model trial, the model begins with its current focus on the primary task using the primary-task problem state. When the interruption occurs, the model notes the visual scene change and begins performing the secondary task (tracking in the tracking-task condition and waiting in the notask condition). Meanwhile, the model also initiates the encoding process described in the previous section, with encoding being executed concurrently using threaded cognition. When the interruption ends, the model retrieves the encoded problem state for the primary task and re-focuses on the primary task.

\section{Model Simulations and Results}

We began our comparison of the encoding strategies by taking the above model of the Monk et al. experiment, integrated with each of the five candidate encoding models, and running simulations to estimate best values of $n$ for the strategies S2-S4. Specifically, we ran 12 simulations for each of a range of possible $n$ values and determined which value produced the best-fitting results (in terms of correlation and RMS error) to those of the original experiment. For S5 the value of $n$ was taken from earlier work $(n=3$ : Altmann $\&$ Trafton, 2002). Using these values for $n$, we ran a final batch of simulations in the same conditions as the original experiment, namely running 12 simulated participants per condition (tracking vs. no-task), each performing 6 trials per interruption duration $(3,8,13 \mathrm{~s})$. We collected three measures: resumption lag, or the time between the end of interruption and the resumption of focus on the primary task; retrieval time, or the time to retrieve the problem state after interruption; and tracking error, or the root-mean-squared error between the target and the cursor over one tracking trial. For resumption lag, note that the model did not predict time between primary-task resumption and the first observable action for this task (as in the original study); we thus estimated this time as a constant to be added to all resumption-lag values for each strategy. Default values were used for all ACT-R parameters as well as those for time estimation and eye movements (see Salvucci, 2001), with the memory decay parameter (base-level learning) set to the standard value (0.5).

Figure 3 shows the resumption lag by interruption duration for each strategy. All strategies exhibit a basic effect 
between the tracking and no-task conditions such that resumption takes roughly 400-500 ms longer after a trackingtask interruption. A large component of this effect is due to two sources: interruptions occasionally end in the middle of a tracking movement, thus taking longer to respond to the change in the tracking condition; and after a tracking interruption, a large eye movement is required to bring visual focus back to the primary task (in contrast to keeping the eyes near the primary task during the no-task interruption).

Along with this basic effect, we can observe some subtle differences in the effect of interruption duration for the various strategies. S1 does not show an increase in resumption lag with an increase in interruption duration; in fact, there is a slight trend toward a decrease in resumption lag. Because this strategy performs encoding throughout the interruption, the problem representation can be very easily retrieved after any delay (retrieval times of roughly $50 \mathrm{~ms}$ or less). S5 shows the opposite trend: Because the strategy performs only 3 retrievals for encoding, the problem representation decays more severely, and the effect of interruption duration is much larger than that observed in the empirical study. S2, S3, and S4, on the other hand, all exhibit behavior that matches fairly well to the empirical results, performing roughly 6 to 13 retrievals for encoding and showing a nominal but clear increase in resumption lag with increase in interruption duration.

Along with the resumption-lag results, we analyzed tracking performance during the tracking-task interruptions. Figure 4(a) shows tracking error for the three experiments in Monk et al. (2008). In all three experiments, the 3-second interruption resulted in the largest tracking error; however, they found that the 3-second interruption error was significantly higher only in Experiment 1, without finding this effect in Experiments 2 and 3. Figure 4(b) shows the model's tracking error for each of the five strategies. S1 exhibited slightly elevated and constant error across all interruption durations due to slight but continual interference from the encoding process. S4 and S5 also exhibited constant error, though slightly lower than $\mathrm{S} 1$ since they experienced no interference from encoding. In contrast, S2 and S3, which experience interference from encoding in the first few seconds of interruption, exhibited slightly higher error for the 3-second interruption. Pairwise tests revealed a significant difference, $p<.05$, between the 3 -second and the other durations for both strategies. While certainly not conclusive, these results seem to suggest that $\mathrm{S} 2$ and $\mathrm{S} 3$ provide a more accurate depiction of tracking performance than the other strategies - that is, people seem to perform encoding in the early stages of interruption rather than wholly before the interruption.

\section{CONCLUSIONS}

Memory processes are critical to the suspension and resumption of tasks in the course of interruption, and an integral component of this processing lies in encoding, or repeated rehearsal, of task-relevant information. In the context of a cognitive architecture such as ACT-R, the representation of memory rehearsal itself is a reasonably straightforward one; however, issues concerning the timing of rehearsal and how it

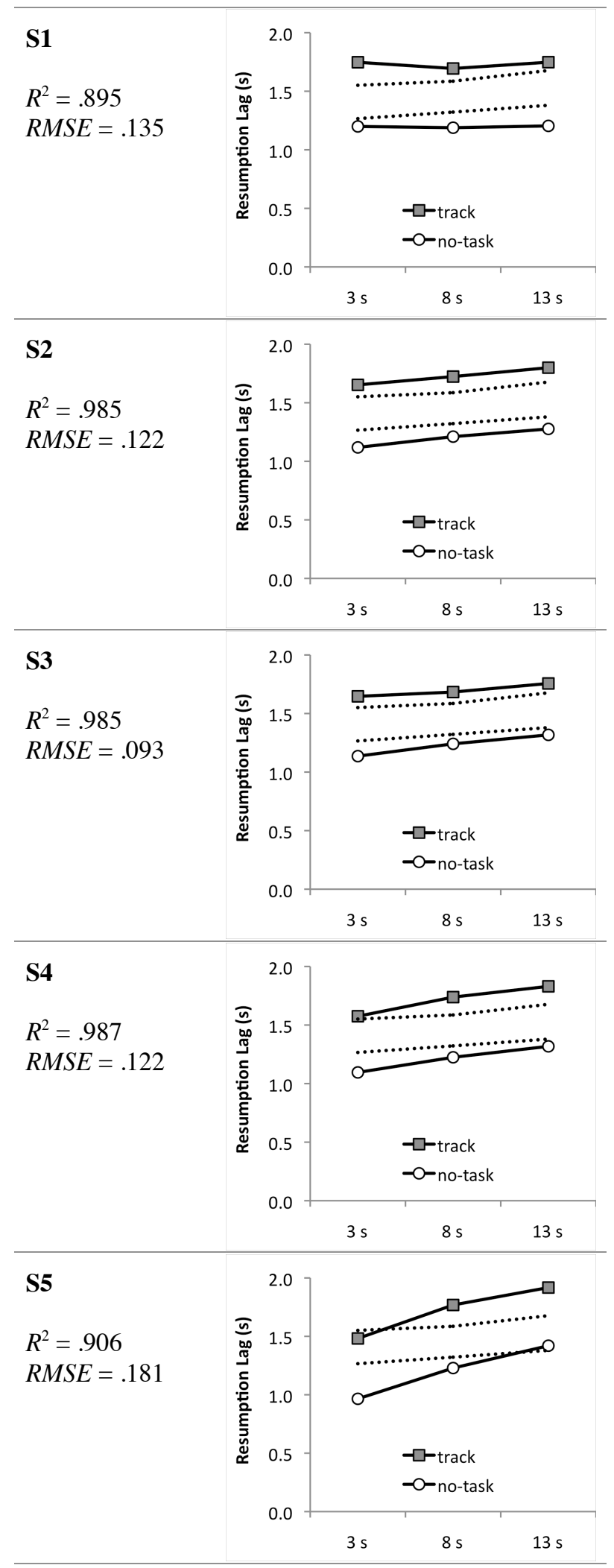

Figure 3: Resumption lag by interruption duration $(3,8$, or $13 \mathrm{~s})$ for each strategy. Dotted lines represent Experiment 3 results from Monk et al. (2008). 

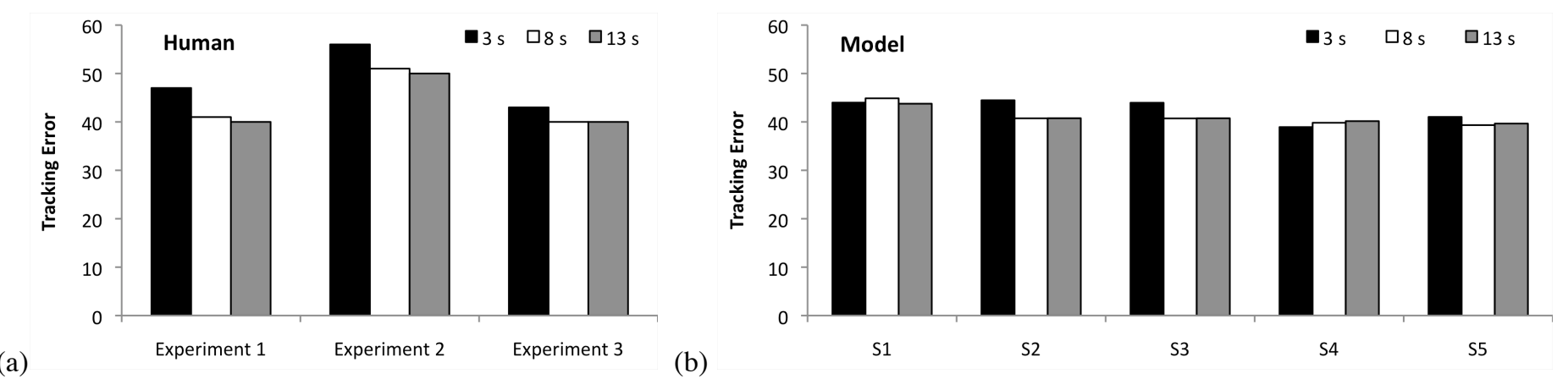

Figure 4: Tracking error as a function of interruption duration for (a) human participants in Monk et al. (2008), and (b) model simulations for each encoding strategy S1-S5.

may be interleaved with concurrent tasks are much less clear. Our work here supports the core ideas of memory for goals theory, namely that behavior during interruption can be well represented as a process of encoding and retrieval of problem state. In addition, the results suggest that encoding occurs for a short period in the early stages of interruption, without continuing throughout the interruption even (surprisingly) during a no-task interruption. These results are consistent with the finding that people do seem to rehearse primary-task information during an interruption (Trafton et al., 2003). The results also support recent work (Salvucci \& Beltowska, 2008) in finding that memory processes may have slight but noticeable effects on secondary-task performance, even for tasks with minimal resource overlap with encoding.

\section{ACKNOWLEDGMENTS}

This work was supported in part by ONR grant \#N0001409-1-0096 and NSF grant \#IIS-0426674.

\section{REFERENCES}

Altmann, E.M. \& Gray, W.D. (2008). An integrated model of cognitive control in task switching. Psychological Review, 115, 602-639.

Altmann, E.M., \& Trafton, J.G. (2002). Memory for goals: An activation-based model. Cognitive Science, 26, 39-83.

Altmann, E.M., \& Trafton, J.G. (2007). Timecourse of recovery from interruption: Data and a model. Psychological Bulletin \& Review, 14, 1079-1084.

Anderson, J.R., Bothell, D., Byrne, M. D., Douglass, S., Lebiere, C., \& Qin, Y. (2004). An integrated theory of the mind. Psychological Review, 111, 1036-1060.

Anonymous (-). Suppressed for blind review.

Bailey, B.P., \& Iqbal, S.T. (2008). Understanding changes in mental workload during execution of goal-directed tasks and its application for interruption management. ACM Transactions on Human-Computer Interaction, 14, 1-28.

Borst, J.P., \& Taatgen, N.A. (2007). The costs of multitasking in threaded cognition. In Proceedings of the Eighth International Conference on Cognitive Modeling (pp. 133-138).
Chisholm, C.D., Dornfeld, A.M., Nelson, D.R., \& Cordell, W.H. (2001). Work interrupted: A comparison of workplace interruptions in emergency departments and primary care offices. Annals of Emergency Medicine, 38, 146-151.

Cutrell, E.B., Czerwinski, M., \& Horvitz, E. (2000). Effects of instant messaging interruptions on computing tasks. In Proceedings of CHI 2000 Extended Abstracts (pp. 99-100).

Dismukes K., Young K. \& Sumwalt R. (1998). Cockpit interruptions and distractions: Effective management requires a careful balancing act. ASRS Directline, 10, 4-9.

Hodgetts, H.M., \& Jones, D.M. (2006). Interruption of the tower of London task: Support for a goal-activation approach. Journal of Experimental Psychology: General, 135, 103-115.

Latorella, K.A. (1999). Investigating Interruptions: Implications for Flightdeck Performance. Technical Report NASA/TM-1999209707, NASA, Washington, DC.

Li, S.Y.W., Cox, A.L., Blandford, A., Cairns, P., Young, R. M., \& Abeles, A. (2006). Further investigations into post-completion error: The effects of interruption position and duration. In Proceedings of the 28th Annual Meeting of the Cognitive Science Conference (pp. 471-476).

Monk, C.A., Boehm-Davis, D.A. \& Trafton, J.G. (2004). Recovering from interruptions: Implications for driver distraction research. Human Factors, 46, 650-663.

Monk, C.A., Trafton, J.G., \& Boehm-Davis, D.A. (2008). The effect of interruption duration and demand on resuming suspended goals. Journal of Experimental Psychology: Applied, 14, 299-313.

Salvucci, D.D. (2001). An integrated model of eye movements and visual encoding. Cognitive Systems Research, 1, 201-220.

Salvucci, D.D., \& Beltowska, J. (2008). Effects of memory rehearsal on driver performance: Experiment and theoretical account. Human Factors, 50, 834-844.

Salvucci, D.D., \& Taatgen, N.A. (2008). Threaded cognition: An integrated theory of concurrent multitasking. Psychological Review, 115, 101-130.

Taatgen, N.A., Rijn, H. v., \& Anderson, J.R. (2007). An integrated theory of prospective time interval estimation: The role of cognition, attention and learning. Psychological Review, 114, 577598.

Trafton, J.G., Altmann, E.M., Brock, D.P., \& Mintz, F.E. (2003). Preparing to resume an interrupted task: Effects of prospective goal encoding and retrospective rehearsal. International Journal of Human-Computer Studies, 58, 583-603. 